\title{
Untersuchung der kapillaren Transportwege im Weißtannenholz
}

\author{
K. Richter und J. Sell \\ Abteilung Holz der EMPA, CH-8600 Dübendorf, Schweiz
}

\begin{abstract}
Die Transportwege für Flüssigkeiten in Weißtannenholz werden nach einem Kapillarsaugversuch mit gefärbtem Wasser und einer Vakuum-Druck-Imprägnierung $\mathrm{mit}$ fluoreszierend eingestelltem EP-Harz mikroskopisch untersucht. Primäre Eindringwege sind die Längstracheiden; die sie verbindenden Hoftüpfel sind die Strukturen, die die Tränkergebnisse dominant bestimmen. Über die Holzstrahlen findet dagegen kein wesentlicher Flüssigkeitstransport statt; normales Kernholz ist weitgehend impermeabel. Im Splint wird die Flüssigkeit hauptsächlich in den Spätholzzonen geleitet, während im Frühholz nur einzelne Zellgruppen zum Transport beitragen. Das deutet an, daß die Tüpfelaspiration selbst innerhalb eines Jahrrings kein einheitlich ausgeprägtes Phänomen ist, und die Permeabilität einer Holzprobe variiert mit dem Ausmaß des Tüpfelverschlußes. Die allgemein bessere Imprägnierbarkeit der Weißtanne im Vergleich zur Fichte kann z. T. mit dem Anteil an Naßkernholz erklärt werden. Zusätzlich jedoch scheint der Tüpfelverschluß weniger intensiv ausgeprägt, weshalb Tannensortimente bei der industriellen Kesseldruckimprägnierung mit einem höheren Tränkdruck gefahren werden sollten als Fichte, um die Eindringtiefe zu verbessern. Wegen des offeneren Kapillarsystems ist die Kollapsgefahr geringer als bei Fichte. Eine trennscharfe und rasche Diagnosemethode für die Tränkbarkeit von Weißtannensortimenten vor der Imprägnierung wurde nicht gefunden.
\end{abstract}

\section{Studies on impregnation pathways in white fir (Abies alba)}

Impregnation pathways in white fir wood were studied based on a capillary rise test with colored water and a vacuum-pressure impregnation with an EP resin contrasted by a fluorescent agent. Microscopic analysis confirmed that primary pathways are the longitudinal tracheids, and structures which influence dominantly all impregnation results are bordered pit pairs. Wood rays are not primarily involved in the transport of impregnation liquids, and normal heartwood is mostly impermeable. In sapwood, liquids extend preferably into the latewood zones, whereas in earlywood only isolated groups of tracheids proved to be permeable. This indicates that pit aspiration is not a homogeneous phenomenon neither within a small sample nor within one tree ring. Consequently, permeability will vary according to the extent of pit aspiration. The gradually better impregnation quality of white fir timber as compared to spruce can be explained only to a certain extent by the existence of wetwood. Additionally, one must assume that pit aspiration in fir is less. This should be used in industrial impregnation by treating fir timber with a somewhat higher pressure to improve impregnation depth, compared to spruce. Because of the more permeable capillary system, danger of wood collapse is limited. No reliable and quick method has been found to predict the treatability of white fir prior to the treating process.

\footnotetext{
${ }^{1}$ Förderung im Rahmen des Nationalen Forschungsprogramms Holz, NFP $12+$, Teil eines Projekts der Abt. Biologie der EMPA St. Gallen (Dr. E. Graf)
}

\section{Einleitung}

Die Wegsamkeit von Hölzern für Flüssigkeiten ist von besonderer Bedeutung für den Erfolg von Imprägnierbehandlungen, den Ablauf von holzchemischen Aufschlußprozessen und auch von Veredelungsmaßnahmen, wie der Beizung und Anstrichbehandlung. Sie wird in entscheidendem Maße von der holzanatomischen Gefügestruktur bestimmt, durch die die natürlichen Eindringwege für flüssige Substanzen vorgegeben sind. Daher wurden im Rahmen eines Forschungsvorhabens über die Ursachen teilweise erheblicher Unterschiede in der Imprägnierqualität von Weißtanne, einer in der Schweiz forst- und holzwirtschaftlich wichtigen Holzart (Sell, Kucera 1989), neben Imprägnierversuchen (Bör 1991) auch mikroskopische Gefügeuntersuchungen durchgeführt. Ziel der Gesamtuntersuchung war es, Hinweise für eine Verbesserung und Vergleichmäßigung der Tränkqualität vor allem bei Tannenschnittholz zu erarbeiten und Ansätze für Diagnosemethoden zu ermitteln, um schlecht imprägnierbare Sortimente gegebenfalls vorzeitig erkennen zu können.

\section{Bisherige Kenntnisse}

\subsection{Anatomie und Wegsamkeit}

Über die holzanatomischen Ursachen der unterschiedlichen Wegsamkeit von Hölzern für Flüssigkeiten liegt ein umfangreiches Schrifttum vor, auf das hier nur zusammenfassend eingegangen werden kann (vgl. hierzu z. B. Siau 1984; Coté 1990). Bei Nadelhölzern, die im vorliegenden Rahmen von vorrangigem Interesse sind, bilden die Lumina der Tracheiden die wichtigsten Leitbahnen für Flüssigkeiten. Den Hoftüpfeln kommt als Verbindungen von Tracheide zu Tracheide für den Flüssigkeitstransport innerhalb des Holzkörpers eine entscheidende Bedeutung zu. Die Tüpfelmembranen weisen im gesamten Kapillarsystem die kleinsten für Flüssigkeiten passierbaren Öffnungen von etwa 0,01 bis $2 \mu \mathrm{m}$ Weite auf. Der Zustand dieser Tüpfelmembranen stellt somit die wichtigste Einflußgröße der Wegsamkeit und Imprägnierbarkeit von Nadelholz dar.

Folgende natürliche Vorgänge können die Permeabilität entscheidend beeinflussen: Zum einen können durch Anlegen der Tüpfelmembranen an die Tüpfelwände während des Wasserentzugs bei der Verkernung oder bei einer 
Trocknung die Tüpfel irreversibel verschlossen werden (Siau 1984). Dieser Effekt tritt vor allem bei den dünnen, nicht sehr steifen Membranen der Frühholzhoftüpfel auf, während die dickeren und darum steiferen Membranen des Spätholzes häufig nicht aspirieren; deshalb ist Spätholz meist besser tränkbar als Frühholz. Zum anderen können die erwähnten feinen Öffunungen der Tüpfelmembranen bei der Verkernung durch Inkrustationen ganz oder teilweise verschlossen werden, wodurch die meist schlechte Tränkbarkeit des Kerns gegenüber dem Splint erklärbar ist (Bailey, Preston 1969).

Einige Autoren machen die unterschiedliche Wegsamkeit der Holzstrahlenzellen sowie die Verbindungswege zwischen ihnen und den angrenzenden Längstracheiden via die sehr verschiedenartige Tüpfelung im Kreuzungsfeld für die großen Unterschiede der Tränkbarkeit der Hölzer verantwortlich (vgl. Baines, Saur 1985). Insbesondere der Anteil und die Permeabilität der Holzstrahltracheiden scheinen für den Flüssigkeitstransport durch die Holzstrahlen von Bedeutung zu sein, während das Holzstrahlparenchym nicht wesentlich beteiligt ist (z. B. Liese, Bauch 1967).

\subsection{Wegsamkeit von Tannenholz}

Die meisten Untersuchungen zur Permeabilität befassen sich vor allem mit den besonders schwer tränkbaren Hölzern wie Fichte und Douglasie sowie auch mit im Gegensatz dazu besonders gut tränkbaren Hölzern wie Kiefer (Splint). Demgegenüber ist das Tannenholz und im speziellen die Weißtanne - vermutlich wegen der überregional geringeren holzwirtschaftlichen Bedeutung - vergleichsweise wenig untersucht worden. Die verfügbaren Aussagen zum Imprägnierverhalten von Tanne werden meist in einen Zusammenhang mit der Ausprägung von Naßkern gestellt. Arganbright und Wilcox (1969) berichten von einer erhöhten tangentialen Wegsamkeit von Tannenholz mit Naßkern. Willeitner (1973) stellt bei einer Untersuchung von 6 Tannenarten eine große Variationsbreite der Aufnahmemengen fest, wobei sich für die Weißtanne - und hier insbesondere für die Proben mit Naßkern - insgesamt eine überdurchschnittliche Tränkbarkeit abzeichnet. Auch Klein et al. (1979) stellen bei Tannenholz mit Naßkern eine verbesserte Imprägnierbarkeit fest und erklären sie mit einer Bakterienbesiedlung im Tüpfelbereich. Allerdings gibt es auch Untersuchungen, in denen Tannennaßkern keine verbesserte Permeabilität zeigte (Passialis, Tsoumis 1984; Sell et al. 1988).

\subsection{Untersuchungsansätze}

Methodisch wurde bei der Untersuchung der kapillaren Transportwege des Holzes meist so vorgegangen, daß Komponenten von in Lösemittel oder in Wasser gelösten Holzschutzwirkstoffen oder Farbstoffen in Holz nachgewiesen wurden. Manche Untersuchungen stützten sich dabei auf Weiserelemente und -verbindungen, die chemisch analysiert bzw. autoradiographisch oder mit der Mikrosonde nachgewiesen wurden. Viele Arbeiten befassen sich mit der licht- und elektronenmikroskopisch sichtbaren Struktur der Transportwege. Recht selten ist dagegen versucht worden, das Kapillarsystem möglichst direkt durch die Beobachtung der Bewegung von Flüssigkeiten auf diesen Wegen zu untersuchen. So hat Liese bereits (1951) die kapillare Bewegung von Ö1 in der sehr schlecht imprägnierbaren Fichte lichtmikroskopisch beobachtet und dabei die dominante Bedeutung der Passierbarkeit der Hoftüpfel und die geringe Bedeutung der Strahlenzellen für diesen Transport erkannt. Erickson und Balatinecz (1964) imprägnierten Douglasienholz mit monomerem Styrol und konnten nach dessen Aushärtung zwischen gefüllten und nicht gefüllten Zellen unterscheiden. Ähnlich sind Trenard und Gueneau (1984) vorgegangen, indem sie flüssiges Gallium von $50^{\circ} \mathrm{C}$ ins Holz von Kiefer und Weißtanne einbrachten und dann bei Raumtemperatur aushärten ließen. Das Eindringen von fluoreszierend gefärbten Kunststoffmonomeren in japanische Nadel- und Laubhölzer haben Furuno und Goto $(1970$; 1972) untersucht und dabei die bereits geschilderte bevorzugte Flüssigkeitsbewegung im Splintholz und innerhalb der Jahrringe im Spätholz bestätigt, wobei sie aber auch abweichende Feststellungen machten. Da die in den zitierten Studien eingesetzten Imprägniermittel im Vergleich zu Wasser unterschiedliche physikalische Eigenschaften aufweisen, versuchten Pendlebury et al. (1990) den direkten Nachweis des Wassertransports durch Einsatz der Gefriermikroskopie. Die Methode ist jedoch technisch sehr aufwendig und erbrachte keine Verbesserungen zu den Arbeiten, bei denen Kunststoffe eingesetzt worden waren. Einen Vorteil der Imprägnierung mit Kunststoffen haben Smulski und Coté (1984) ausgenutzt, indem sie nach der Aushärtung eines Alkydharzes die Holzsubstanz auflösten und die Negativabdrücke der Flüssigkeitsleitbahnen untersuchen konnten.

Für die Gefügeuntersuchungen im Rahmen dieser Studie wurde neben einem Kapillarsaugversuch mit gefärbtem Wasser eine Imprägnielmethode mit fluoreszierend eingestelltem Epoxidharz eingesetzt, das sich bereits früher bei der Untersuchung des Hohlraumsystems von Baustoffen bewährt hat (Sell 1979).

\section{Material and Methoden}

\subsection{Kapillare Saugfähigkeit}

Zur Bestimmung der kapillaren Saugfähigkeit wurden 72 Probestäbe aus vier Stammhöhen einer Tanne mit quadratischem Querschnitt $\left(4,5 \times 4,5 \mathrm{~cm}^{2}\right)$ und einer Länge in Faserrichtung von $20 \mathrm{~cm}$ geschnitten. Nach ihrer Auskonditionierung im Normalklima $\left(20^{\circ} \mathrm{C} / 65 \% \mathrm{r}\right.$. L.) wurden die Probestäbe gewogen und anschließend mit einer Hirnfläche $1 \mathrm{~cm}$ tief in eine $0,3 \%$ ige wäßrige Lösung eines sauren Textilfarbstoffes gestellt (Neolan Glaucin E-A 235\%; Ciba Geigy). Dieses blaue Triphenylmethan hat ein Molekulargewicht von 749 und eine Molekülgröße von ca. $170 \ldots 200 \mathrm{~nm}$ Länge und $120 \ldots 150 \mathrm{~nm}$ Breite.

Zur Bestimmung der Gewichtzunahme wurden die Proben nach 1, 3, 6, 9 und 24 Stunden aus dem Gefäß genommen und nach dem Abtropfen überschüssigen Wassers gewogen; aus der jeweiligen Differenz zum Anfangsgewicht wurde die kapillar aufgesaugte Wassermenge in Gramm berechnet. Nach Abschluß des Versuches wurden die Proben diagonal aufgeschnitten und die auf den Schnittflächen erkennbaren gefärbten Zonen visuell begutachtet (insbesondere hinsichtlich verschiedener Steighöhen im Kern- bzw. Splintbereich oder sonstiger auffälliger Besonderheiten). Zur Beurteilung der Abnahme der gefärbten Holzanteile mit der Steighöhe 
wurden ausgesuchte Proben horizontal schichtenweise aufgeschnitten. Besonders gut saugende Gewebepartien wurden lichtmikroskopisch untersucht.

\subsection{Vakuum-Druck-Imprägnierung mit einem angefärbten Epoxidharz}

Ziel dieser Untersuchungsmethode war es, die Transportwege einer Tränkflüssigkeit gewissermaßen in situ für die Licht- und Rasterelektronenmikroskopie kontrastreich darzustellen. Hierzu wurden im Normalklima auskonditionierte Tannenproben $\left(4 \times 4 \times 8 \mathrm{~cm}^{3}\right)$ mit einem sehr niedrigviskosen, aliphatischen Epoxidharz (Araldit DY 026, Ciba Geigy) imprägniert, dem ein cycloaliphatischer Polyaminhärter (HY 2962, Ciba Geigy) im Verhältnis 70:30 zugemischt wurde. Zur Kontrastierung wurde ein Fluoreszenz-Farbstoff (z. B. Fluorol Gelb 088, BASF) in einer Dosierung von $0,05 \%$ (bezogen auf die fertige Mischung) beigegeben ${ }^{2}$. Der Farbstoff hat ein Molekulargewicht von 296, eine Molekülgröße von 100 ... $130 \mathrm{~nm}$ Länge und 75 ... $90 \mathrm{~nm}$ Breite. Die Viskosität des Harzes unmittelbar nach der Mischung beträgt $12 \mathrm{mPa} s$ und steigt nach einer Stunde bei $20^{\circ} \mathrm{C}$ auf $20 \mathrm{mPa}$ s, nach 2 Stunden auf ca. $25 \mathrm{mPa}$ s an. In Vorversuchen mit Membranfiltern aus Celluloseacetat (Millipore, $\mathrm{d}=5 \mathrm{~cm}$; Wasserstrahlvakuum ca. $20 \mathrm{mbar}$ ) durchfloß das Harzgemisch Filter der Porengröße $150 \mathrm{~nm}$ noch 4 Stunden nach Ansatz der Mischung. Filter der Porengröße $<50 \mathrm{~nm}$ wurden dagegen auch von frisch angemischtem Harz nicht mehr durchflossen.

Die Hirnflächen der zu imprägnierenden Hölzer wurden mit Aluminiumfolie abgedichtet. Für orientierende Untersuchungen wurden, um eine Haupteindringrichtung vorzugeben, zudem alternativ die Radial- und Tangentialflächen der Proben abgeklebt.

Der Tränkvorgang umfaßt die folgenden Schritte:

1. $2 \mathrm{~h}$ Evakuierung der Probenklötzchen in einem Exsikkator bei 0.6 bis 0.9 mbar.

2. Anrühren der Harzmischung und unverzügliches Fluten der Probe unter Vakuum.

3. $15 \mathrm{~min}$ Evakuation des gefluteten Behälters bei 1,3 mbar.

4. $2 \mathrm{~h}$ Druckbehandlung der gefluteten Probe bei einem Druck von 10 bar.

5. 1- bis 2tägige Aushärtung des Harzes bei Zimmertemperatur.

Unmittelbar nach der Aushärtungsphase wurden aus den mittleren Bereichen der Proben ca. $1 \mathrm{~cm}$ dicke Scheiben herausgesägt und nach Glättung der Querschnittsflächen die Ausbreitung des eingedrungenen Harzes beurteilt. Von ausgesuchten Zonen wurden mit speziell harten Mikrotomklingen Dünnschnitte für die UVFluoreszenzmikroskopie hergestellt. Parallel dazu wurden Präparate zur Untersuchung mit dem Rasterelektronenmikroskop angefertigt. Da das verwendete EP-Harz sehr säureresistent ist, konnte die Holzsubstanz - nach Aushärtung des Harzes in den Lumina - mit konzentrierter Schwefelsäure (72\%ig) hydrolysiert werden. Zurück blieben EP-Abdrücke bzw. -Modelle des Hohlraumgefüges, die im Licht- und vor allem im Rasterelektronenmikroskop untersucht wurden.

2 Das verwendete Epoxidharz kann in flüssigen Zustand allergene Hautreizungen verursachen, weshalb entsprechende Schutzmaßnahmen zu ergreifen sind.
Tabelle 1. Kapillar aufgesaugte Wassermenge

\begin{tabular}{lllllll}
\hline Stammhöhe (m) & $n$ & $\bar{x}$ & $s$ & Minimum & Maximum \\
\hline $1-4$ & & 23 & 14,35 & 2,49 & 11,15 & 20,73 \\
$5-8$ & 19 & 13,88 & 3,39 & 9,48 & 19,88 \\
$9-12$ & 17 & 14,28 & 2,87 & 9,78 & 20,43 \\
$13-16$ & 13 & 15,35 & 2,83 & 11,98 & 20,32 \\
\hline
\end{tabular}

$n$ Anzahl Stichproben, $\bar{x}$ Mittelwert, $s$ Standardabw.

\section{Ergebnisse}

\subsection{Kapillare Saugfähigkeit}

Die Wasseraufnahme nach 24 Stunden ist, unterteilt in die 4 Stammhöhenbereiche, in Tabelle 1 zusammengefaßt. Die Meßwerte belegen, daß das mittlere Saugverhalten des Tannenholzes über die Stammhöhe nur wenig variiert. Auch die Berücksichtigung der Werte aus den Zwischenwägungen bestätigt diese Aussage (Bild 1). Der Verlauf der Kurven macht deutlich, daß die Wasseraufnahme nach 24 Stunden nicht abgeschlossen ist. Aus den Einzelwerten ist eine ausreichende Differenzierung zwischen schlecht und gut saugenden Proben frühestens nach 6 Stunden möglich; nach 24stündiger Versuchsdauer ergeben sich sichere Unterscheidungen.

Im Hinblick auf die Eignung der Methode zur Kennzeichnung der Tränkbarkeit wurden die Wasseraufnahmen mit den Ergebnissen der Imprägniervarianten verglichen, die an Proben aus dem gleichen Stamm durchgeführt worden waren (Bör 1991). Obwohl sich der Vergleich nicht auf völlig identische Querschnitte bezieht, konnten bei $68 \%$ der Proben Zusammenhänge zwischen der kapillaren Aufsaugmenge und der Schutzmittelaufnahme nach Kesseldrucktränkung nachgewiesen werden. Aufgrund dieser Hinweise wurde die Eignung der kapillaren Saugfähigkeit als Indikatormethode in Zusatzversuchen von Bör (1991) quantitativ näher untersucht.

Die Analyse der diagonal aufgeschnittenen Probestäbe bestätigt, daß die Saugfähigkeit des Splintholzes unabhängig von der jeweiligen Stammhöhe bei der untersuchten Tanne deutlich besser ist als die des Kernholzes. Mit zunehmender Saughöhe innerhalb der Proben reduzieren sich die gefärbten Bereiche auf einzelne Spätholz-

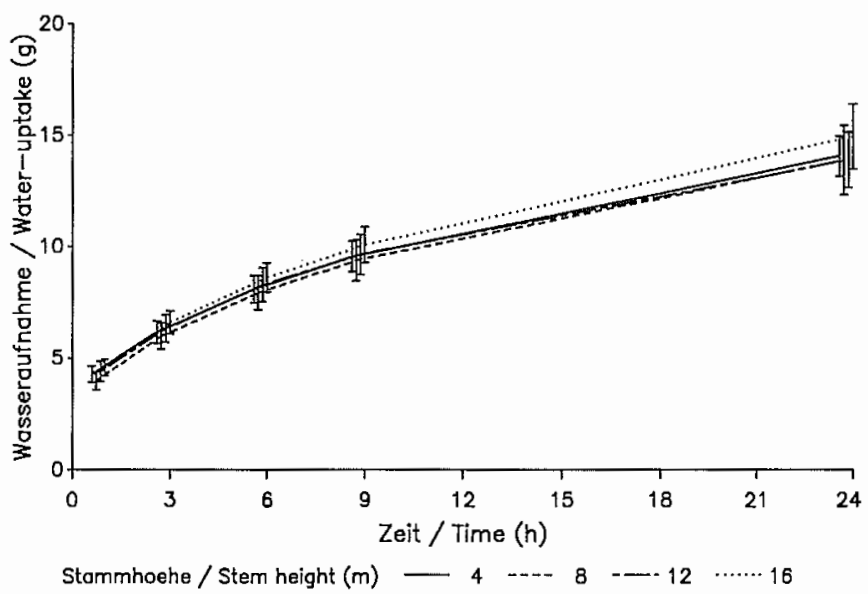

Bild 1. Wasseraufnahme im Kapillarsaugversuch bei versehiedenen Stammhöhen (Mittelwerte mit 95\% Vertrauensbereich)

Fig. 1. Water uptake in capillary tise test at different heights of the stem (average with 95 percent confidential interval) 


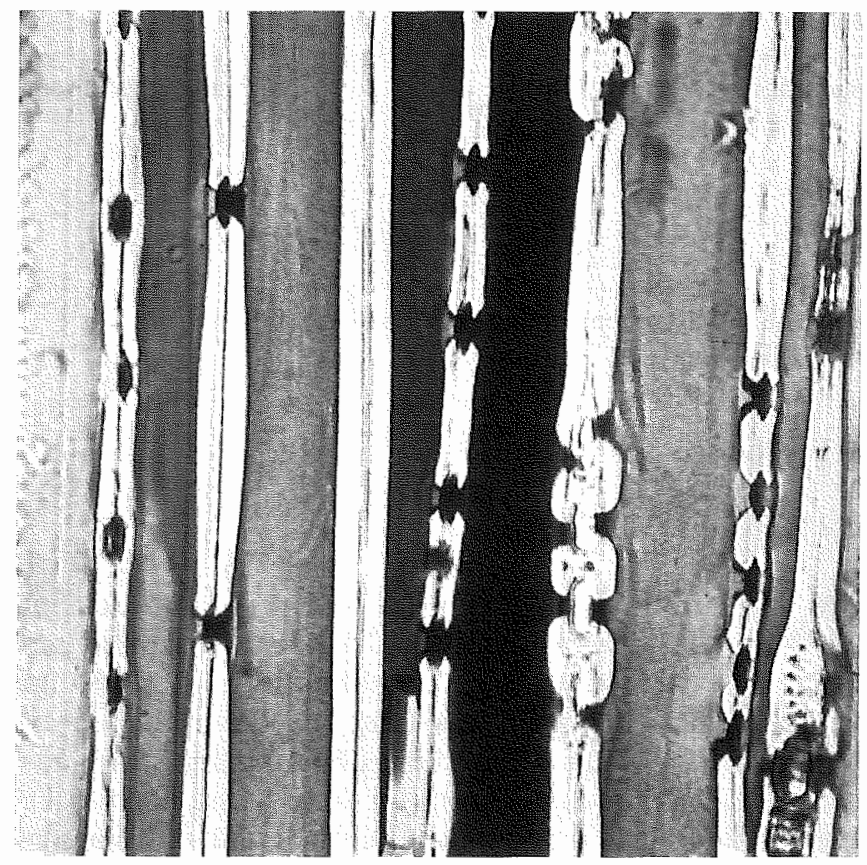

Bild 2. Gradienten der Farbstoffkonzentration zwischen benachbarten Längstracheiden. Tangentialschnitt, $400 \times$

Fig. 2. Gradients in coloration intensity between neighboring tracheids (TS, $400 \times$ )

bänder, die vom umgebenden Gewebe deutlich abgegrenzt sind. In einzelnen Spätholzzellen dieser Bänder ist Farbsubstanz zu erkennen, die auf der Zellwand abgelagert ist; in die Zellwand selbst dringen die Farbstoffmoleküle nicht ein. Auch in Längsschnitten sind deutliche Farbgradienten zwischen benachbarten Tracheiden erkennbar (Bild 2). Die Akkumulation von

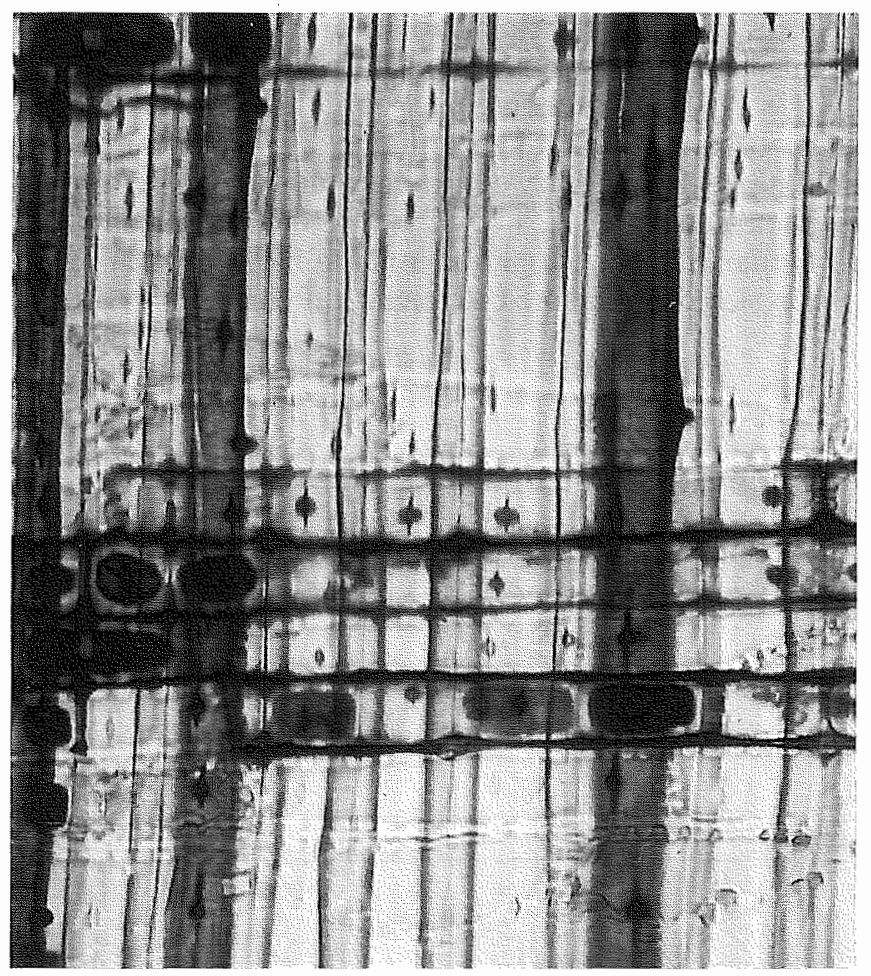

Bild 3. Kreuzungsfeld mit Farbgradienten zwischen Tracheiden und Holzstahlparenchym $(400 \times)$

Fig. 3. Cross-field section showing color gradients between tracheids and ray parenchyma $(400 \times)$
Farbstoffmolekülen in den Kammern der Hoftüpfel belegt, daß die Membranporen Engpässe darstellen, an denen größere Farbstoffmoleküle beim Passieren ausgefiltert werden (Chromatogramm-Effekt). Für die halbbehöften Tüpfel zwischen Tracheiden und Holzstrahlparenchym gilt Entsprechendes. Aus den Abstufungen in der Farbintensität zwischen längs- und quergerichteten Zellen ist ersichtlich, daß die Flüssigkeitsbewegung beim kapillaren Transport in der Regel von den Längstracheiden in die Holzstrahlzellen verläuft und nicht umgekehrt (Bild 3). Die innerständigen Holzstrahlparenchymzellen weisen im Vergleich zu den Tracheiden stets eine geringere Verfärbungsintensität auf. Beim Passieren der einseitig behöften Tüpfel zwischen längs- und quergerichteten Zellen werden somit mehr und größere Farbmoleküle ausgefiltert. Randständige Holzstrahlzellen sind nie gefärbt und tragen daher überhaupt nicht zum Flüssigkeitstransport bei.

\subsection{Vakuum-Druck-Imprägnierung mit einem angefärb- ten Epoxidharz}

In Vorversuchen wurde zunächst geprüft, ob das Eindringverhalten des EP-Harzes trotz der unterschiedlichen physikalisch-chemischen Eigenschaften mit dem von wäßrigen Imprägnierflüssigkeiten vergleichbar ist. Dazu wurde an Proben aus im Stamm hintereinanderliegenden Bereichen ein kapillarer Saugversuch bzw. eine Harzimprägnierung durchgeführt. In beiden Proben waren die gleichen Querschnittsflächen im Splintholz gefärbt bzw. imprägniert. Weiter wurde eine Probe mit Naßkern im gleichen Imprägnierprozeß gemeinsam mit naßkernfreiem Tannenholz imprägniert. Die Harzverteilung im Querschnitt läßt für die Probe ohne Naßkern eine gute Eindringung nur im Splint erkennen, während die Naßkernprobe vor allem im Kernholz fast vollständig harzdurchtränkt ist (Bild 4). Nachdem damit die erwarteten Eindringmuster auch bei der Harzimprägnierung bestätigt werden konnten, wurden die Eindringwege des Harzes mikroskopisch näher untersucht.

Im normalen Tannenkernholz breitet sich das Harz vornehmlich in den Spätholzzonen aus. Von den Seitenflächen der Proben verlaufen tangentiale Bänder, in denen die Spätholztracheiden mit Harz gefüllt sind,

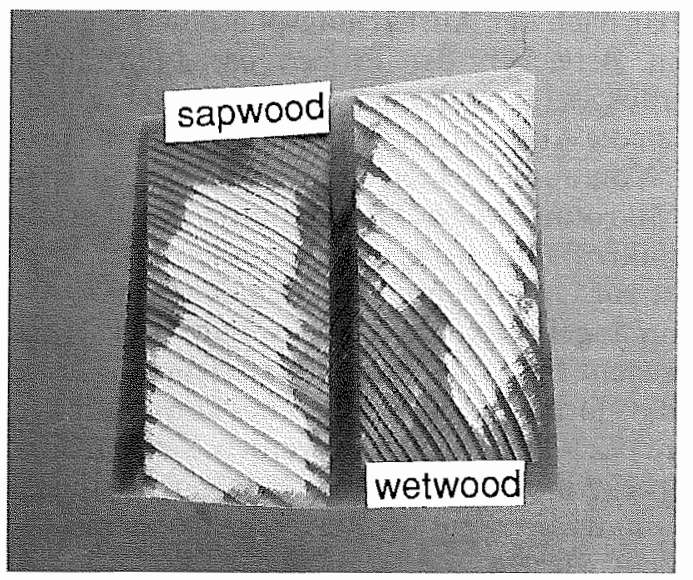

Bild 4. Makroskopische EP-Harzverteilung in normalen Tamnenholz und im Naßkern

Fig. 4. Macroscopic EP resin distribution in normal fir and fir wetwood 


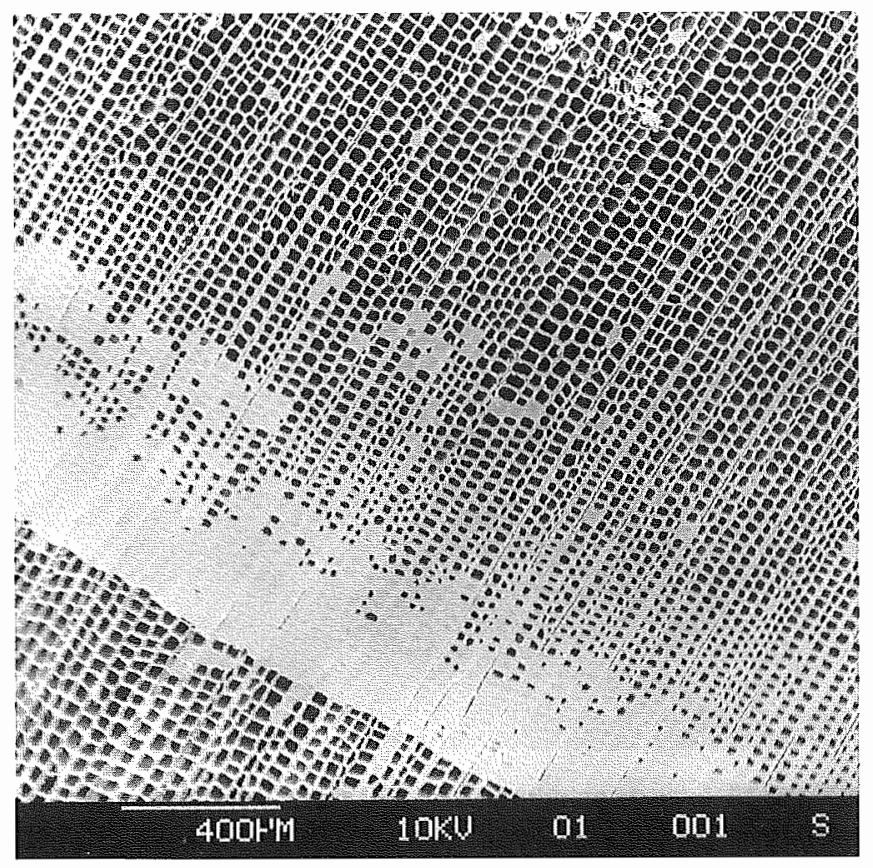

Bild 5. Mit EP-Harz imprägnierte, bandförmige Spätholzzone neben nichtdurchtränktem Frühholz. REM quer

Fig. 5. EP-impregnated latewood and impermeable earlywood (SEM, CS)

während die angrenzenden Frühholzzonen desselben bzw. des nächsten Jahrrings nicht imprägniert sind (Bild 5). Hiervon abweichende Muster zeigen Splintholz und Naßkernholz, wo auch die Frühholzzellen überwiegend gefüllt sind.

Neben zusammenhängenden harzgefüllten Zonen gibt es Bereiche (,Nester'), in denen nur wenige Zellen sepa-

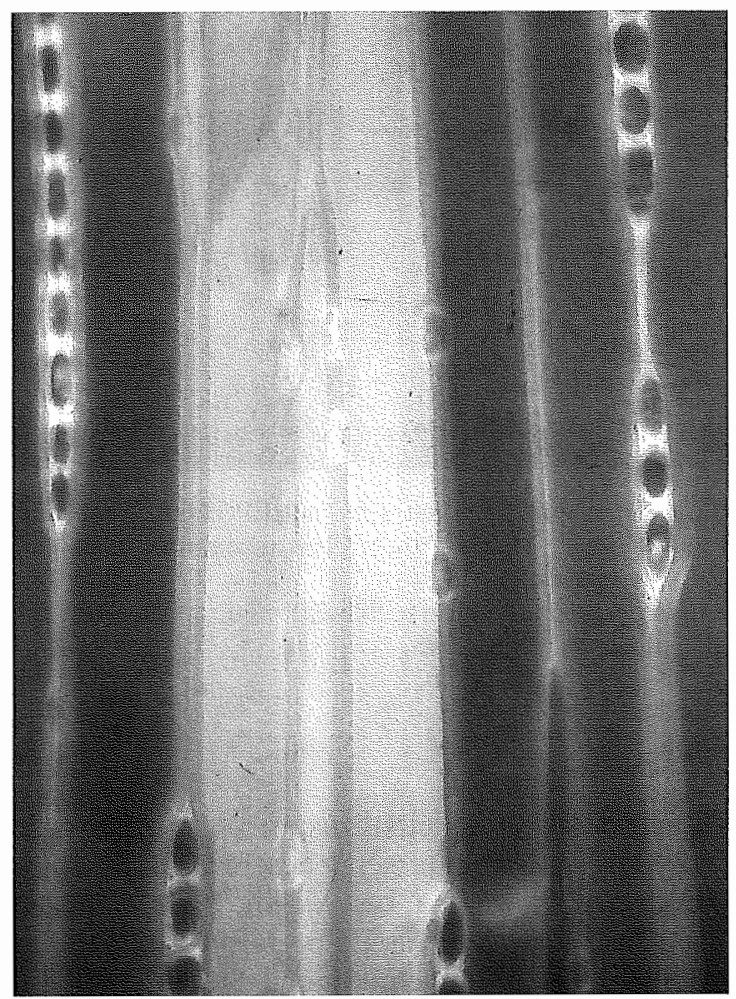

Bild 6. Beispiel einzelner harzgefüllter Tracheiden im Frühholz. Tangentialschnitt $(350 \times)$

Fig. 6. Example of some resin filled earlywood tracheids (TS, $350 \times$ )

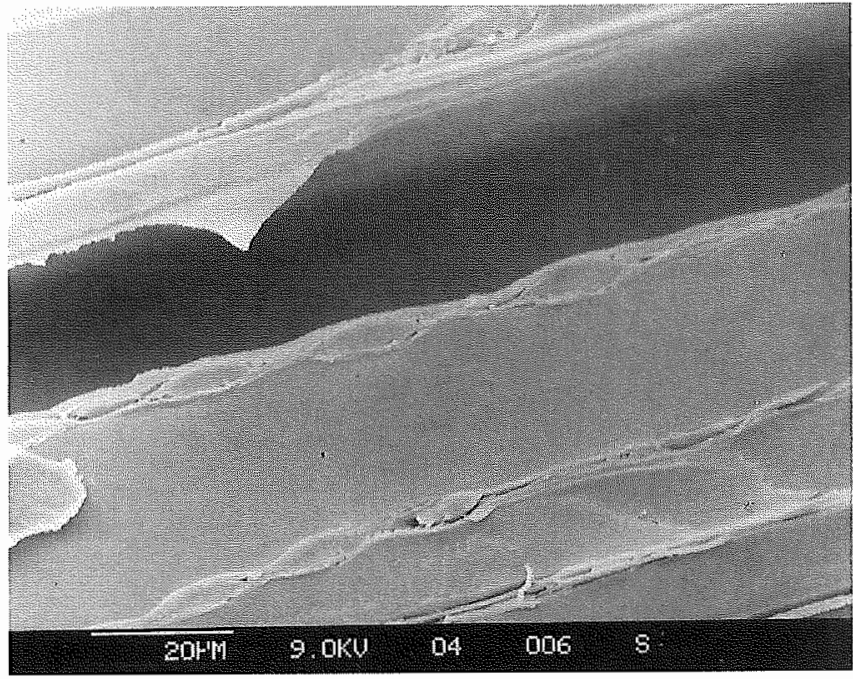

Bild 7. Aspirierte Hoftüpfel mit EP-harzgefüllter Tüpfelkammer und angrenzender nicht gefüllter Tracheide (Pfeile). REM tangential

Fig. 7. Aspirated bordered pits with EP-resin filled pit chambers adjacent to an empty tracheid (arrows) (SEM, TS)

riert im sonst nichtgetränkten Holz fluoreszieren (Bild 6). Im Vergleich zum umgebenden Gewebe müssen diese Zellen über passierbare Tüpfelverbindungen verfügen, während die Verbindungswege zu den nebenliegenden Tracheiden verschlossen sind. Dieser Befund wird durch EP-harzgefüllte Tüpfelkammern mit aspiriertem Torus bestätigt, die einen Durchfluß von der gefüllten in die leere Tracheide verhindern (Bild 7). Die dominante Bedeutung der Hoftüpfel für den Flüssigkeitstransport ist auch aus einer z. T. erhöhten Fluoreszenz der Tüpfelmembranen ableitbar. Die fluoreszierenden Tüpfel von Bild 8 waren für das Harz passierbar, jedoch wurden gewisse Anteile von Farbstoff an den Tüpfelmembranen zurückgehalten. Bild 9 zeigt EP-Harz, das unmittelbar nach dem Passieren eines Tüpfels auspolymerisiert ist.

Die Holzstrahlen des Tannenholzes werden auch vom im Vakuum-Druckverfahren eingebrachten EP-Harz

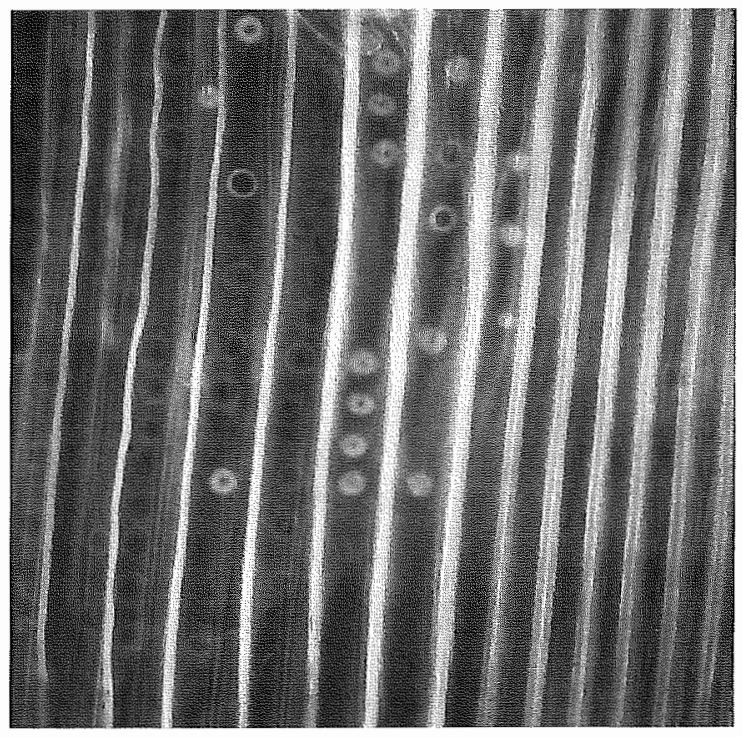

Bild 8. Anhäufung von Fluoreszenzfarbstoff an den Tüpfelmembranen der Hoftüpfel. Tangentialschnitt $(170 \times)$

Fig. 8. Accumulation of fluorescent dye on pit membranes of bordered pits (TS, $170 \times$ ) 


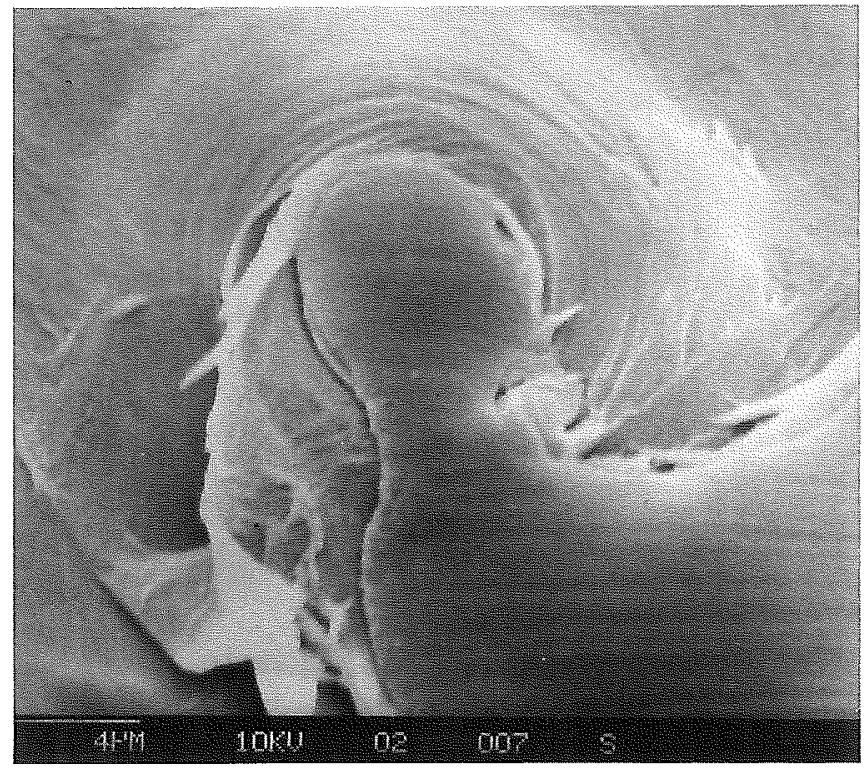

Bild 9. Auspolymerisiertes EP-Harz nach Durchfluß der Tüpfelöffnung, Holz mit Schwefelsäure entfernt. REM, radial

Fig. 9. In situ cured resin after having passed a bordered pit. Wood removed by sulphuric acid. (SEM, RS)

nicht als primäre Eindringwege benutzt. Bild 10 zeigt ein charakteristisches Bild mit harzgefüllten Tracheiden neben vollständig leeren Holzstrahlen. Die Membranen der Tüpfel zwischen Längstracheiden und Strahlen bilden offensichtlich Barrieren, die vom Harz nicht durchflossen werden können. Auch die Abdrücke von Kreuzungsfeldtüpfeln weisen darauf hin, daß das EP-Harz von den Tracheiden kommend jeweils an den Membranen zurückgehalten wurde (Bild 11). Nur in wenigen Ausnahmen, insbesondere im Randbereich der Proben, konnte das EP-Harz auch in den Holzstrahlen nachgewiesen werden (Bild 12).

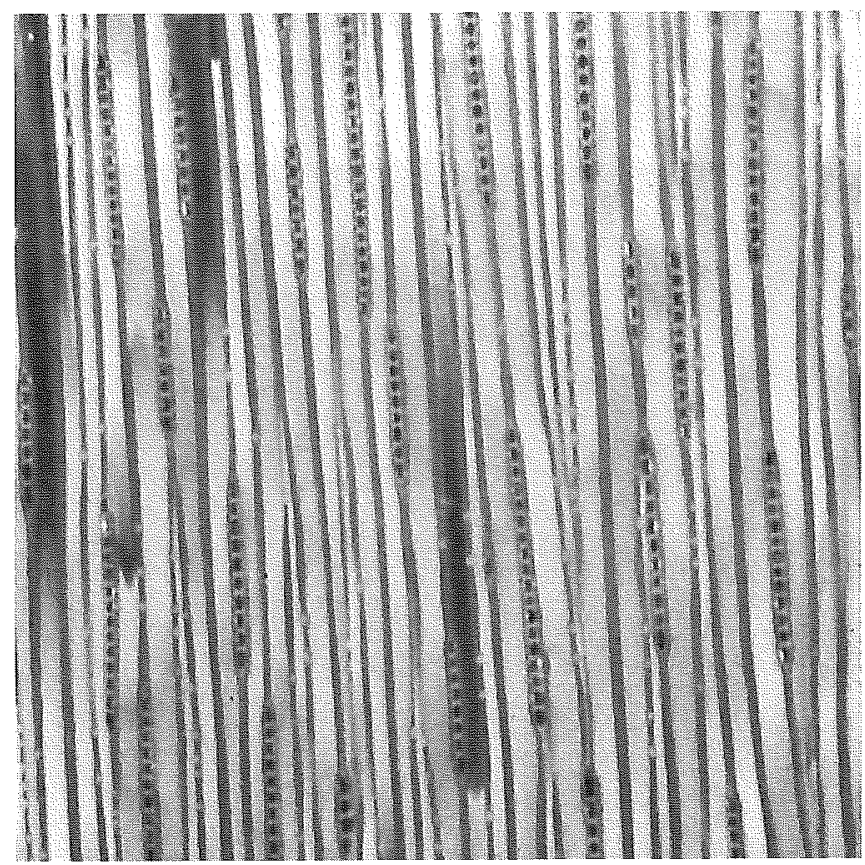

Bild 10. EP-harzgefüllte Splintholztracheiden neben nicht gefüllten Holzstrahlen. Tangentialschnitt $(85 x)$

Fig. 10. EP-resin filled sapwood tracheids next to empty wood rays (TS, $85 \times)$

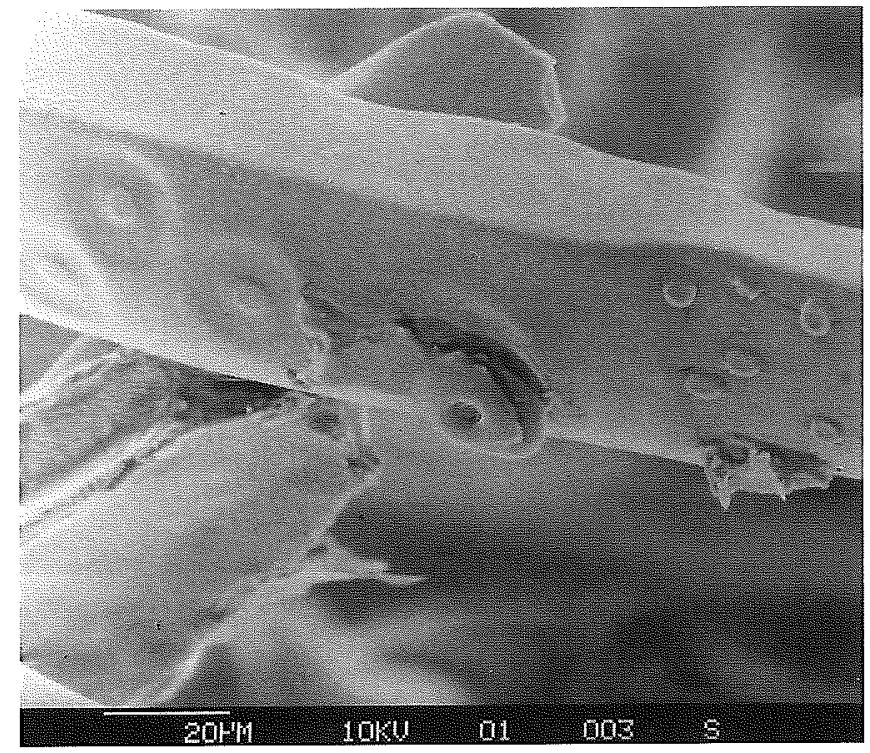

Bild 11. Tüpfel verhindern den EP-Harzfluß von der Tracheide in die Holzstrahlparenchymzelle. Die EP-Harzreplica des Lumens einer Längstracheide mit Kreuzungsfeld (rechts im Bild) zeigt Harzabdrücke der nicht passierbaren Kreuzungsfeldtüpfel. Von den angrenzenden Hoftüpfeln war nur einer für das EP-Harz passierbar (Pfeil). REM quer

Fig. 11. Pits are blocking the passage of EP resin from a tracheid into a ray parenchyma cell. The EP resin replica of the tracheid lumina with a cross-field (right side) shows resin casts of bulk blocking cross-field pits. Only one of the neighboring bordered pits has been passed by the EP resin (arrow). (SEM, CS)

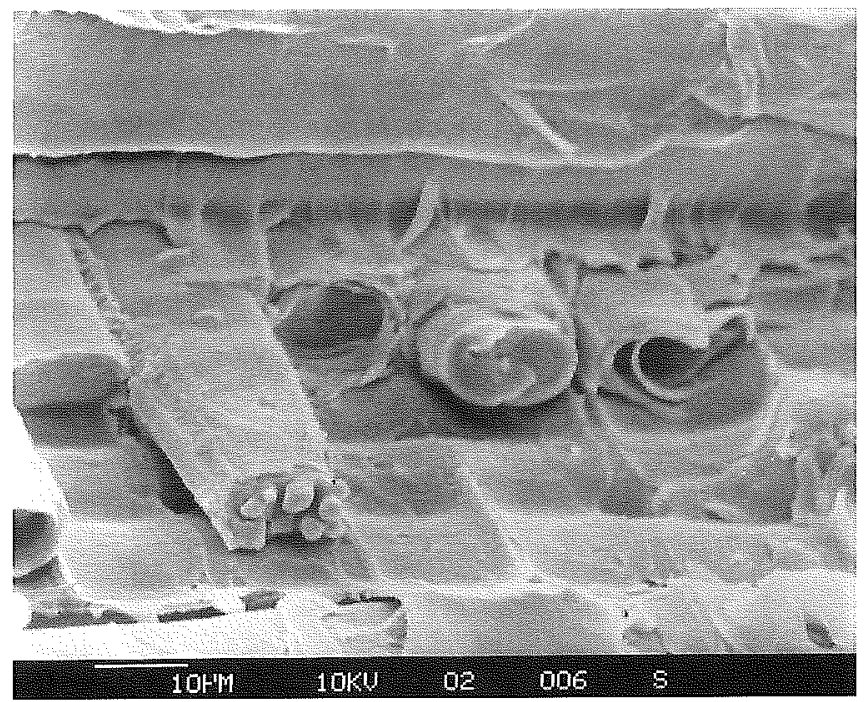

Bild 12. Randbereich einer Tannenprobe mit EP-Harzreplica eines Kreuzungsfelds. Hier sind Holzstrahlenzellen ansnalhmsweise gefüllt gewesen. REM tangential

Fig. 12. Sample peripheral section with a EP resin replica. Ray cells are filled exceptionally. (SEM, TS)

\section{Diskussion}

Die Prinzipien des Flüssigkeitstransportes im Holz sind von Siau (1984) zusammenfassend beschrieben worden. Für Anwendungen, wie sie bei vielen Prozessen der Holzbearbeitung von Interesse sind (Einbringen von Schutzmitteln, Aufschlußchemikalien, Substanzen zur Dimensionsstabilisierung; Verankern von Anstrich- und Klebstoffen etc.), erfolgt der Transport als Massenfluß (,bulk flow'). Eine solche Flüssigkeitsbewegung wird 
über einen statischen oder kapillaren Druckgradienten ausgelöst und setzt ein System zusammenhängender Kapillaren (Kapillarporosität) voraus. Für einen kapillaren Transport müssen diese ausreichend enge Radien besitzen; eine untere Begrenzung des Kapillarradius ergibt sich durch die Molekülgröße des flüssigen Stoffes. Die Flüssigkeit muß zudem eine genügende Oberflächenspannung und Viskosität aufweisen.

All diese Voraussetzungen gelten sowohl für den einfachen Saugversuch mit angefärbtem Wasser als auch für den aufwendigeren Tränkversuch mit angefärbtem EP-Harz unter Vakuum-Druckeinwirkung, wie sie in der vorliegenden Stufe zur Beschreibung des Kapillarsystems der Weißtanne eingesetzt wurden. Es entspricht den Erwartungen, daß beide Methoden grundsätzlich ähnliche Ergebnisse erbrachten. In Übereinstimmung mit zahlreichen früheren Untersuchungen an Nadelhölzern (Liese 1951; Bailey 1958; Buro, Buro 1959; Bailey, Preston 1969) konnte auch für Weißtanne nachgewiesen werden, daß die Längstracheiden bzw. der Zustand der sie verbindenden Hoftüpfel für den Flüssigkeitstransport von dominanter Bedeutung sind. Die Beobachtung, daß Flüssigkeit im Splintholz besser in den Spätholzzellen geleitet wird, bekräftigt die Hypothese von Philips (1933), daß die steiferen Membranfibrillen im dickwandigen Spätholz eine Tüpfelaspiration verhindern. Beim Verkernungsprozeß werden dann durch die Ablagerung von Extraktstoffen die Margoporen auch der nicht aspirierten Spätholztüpfel verstopft, weshalb in beiden Versuchsteilen keine Flüssigkeit in normales Kernholz eingebracht werden konnte.

Im Gegensatz zu Kiefer, deren heterogene Holzstrahlen einen wesentlichen Beitrag zur radialen Flüssigkeitsbewegung leisten (Buro, Buro 1959; Wardrop, Davies 1961; Bauch et al. 1983; Trenard, Gueneau 1984), kommt den homogenen Holzstrahlen der Weißtanne nur eine geringe, lokal sogar keine Bedeutung als Transportwege für Imprägnierflüssigkeiten zu. Dies belegen auch die Befunde von Trenard und Gueneau (1984), die für Tanne nach einer Galliumimprägnierung bei 16 bar neben vollständig gefüllten Tracheiden nur wenige Tropfen des Metalls in den Holzstrahlzellen nachwiesen. Die geschlossenen Tüpfelmembranen zwischen Längstracheiden und Strahlparenchym bilden somit natürliche Barrieren, die von Flüssigkeiten im Massenfluß nicht durchdrungen werden können. Dies konnte insbesondere mit den EP-Harzabdrücken nachgewiesen werden (Bild 11). Damit scheidet der bei Tannenholz höhere Raumanteil von Holzstrahlen (6 ... 10\%) im gesamten Zellsystem als Erklärungsgrund für die bessere Imprägnierbarkeit von Weißtanne gegenüber Fichte $(5 \ldots 7 \%)$ aus.

Bör (1991) nennt die Ausprägung von Naßkernholz als entscheidenden Grund für die in seinen Untersuchungen festgestellten höheren Aufnahmemengen bei Tanne vs. Fichte. Auch Willeitner (1973), Arganbright und Wilcox (1969) sowie Klein et al. (1979) berichten von verbesserter Wegsamkeit im Naßkernholz bei Tanne. Wir konnten dies an Naßkernproben aus dem Material von Bör bestätigen. Parallelversuche mit Naßkernholz aus einer Untersuchung von Sell et al. (1988), in die mittels Druckimprägnierung keine erhöhten Schutzmittelmengen eingebracht werden konnten, blieben auch mit den hier eingesetzten Methoden negativ. Da die erhöhte Wegsamkeit des Tannennaßkerns in vielen Studien im Zusammenhang mit einem Bakterienbefall erklärt wird (z. B. Klein et al. 1979), wie er für den sogenannten ,pathologischen' Naßkern charakteristisch ist (Schütt 1981; Brill et al. 1981), beziehen sich die Aussagen zur verbesserten Kernholzpermeabilität offensichtlich nur auf diese Form des Naßkerns. Auch Passialis und Tsoumis (1984) stellen keine erhöhten Aufnahmemengen an Naßkernproben fest, bei denen nachweislich kein Bakterienbefall vorlag.

Trotz eines nicht unerheblichen Anteils von Naßkernbildung bei der Mehrzahl von Schweizer Weißtannen (Sell, Kucera 1989) scheidet dieses Phänomen somit als alleinige Ursache für die verbesserte Imprägnierbarkeit gegenüber Fichtenholz aus. Auch in unseren Versuchen mit normalem Tannenholz war dessen Wegsamkeit substantiell besser als die der mitimprägnierten Fichten. Es kann daraus gefolgert werden, daß der Hoftüpfelverschluß bei Weißtanme generell weniger intensiv ist als bei Fichte. Eine Beeinflussung durch die bei Tanne vorhandene Warzenstruktur scheint möglich, durch die ein enges Anliegen der Margofibrillen an den Tüpfelwulst verhindert wird (Bellmann 1987).

Die für die praktische Holzimprägnierung nutzbare Auswirkung dieser Eigenschaft ist, daß der Imprägnierdruck bei reinen Tannensortimenten höher zu fahren ist als 6 bar, der bei Fichte aufgrund der Kollapsgefahr von Sell et al. 1988 als obere Grenze empfohlen wurde. Mit einer verbesserten Eindringtiefe ist zu rechnen. Dies entspricht auch den schon zuvor zitierten Beobachtungen von Trenard und Gueneau (1984), die erst bei einem erhöhten Druck eine gute Galliumeindringung in Tannenholz erzielten. Aufgrund des offeneren Kapillarsystems bei Tanne ist ein rascherer Druckausgleich möglich und somit die Gefahr von Zellwandeinbrüchen gering. Das dürfte übrigens ein Grund dafür sein, daß in der schweizerischen Praxis bisher keine Zellkollapsschäden an Weißtanne bekannt geworden sind.

Die mit den eingesetzten Methoden erkennbaren Unterschiede in der Flüssigkeitsaufnahme von Splintholzpartien innerhalb einer Probe und selbst innerhalb desselben Jahrrings (Ausprägung von Nestern, Bild 6) belegen, da 3 der Tüpfelverschlu $\beta$ - als das die Permeabilität bestimmende Pliänomen - nicht homogen innerhalb einer Holzprobe ausgeprägt sein muß. Dies wird auch von Coté (1990) bestätigt. Die dadurch mögliche und vielfach festgestellte große Variabilität im Aufnahmeverhalten selbst benachbarter Holzpartien (bei Weißtanne z. B. von Willeitner 1973) macht es schwierig, statistisch trennscharfe Diagnoseverfahren zur Vorherbestimmung des Aufnahmeverhaltens einzusetzen. Das ist mit ein Grund dafür, warum alle bisher geprüften Verfahren (z. B. Arganbright, Wilcox 1969; Venturio Arganbright 1979; Humphrey, Morell 1988) nicht zur Praxisreife gelangten. Zwar ergaben die von Bör (1991) durchgeführten Vergleiche zwischen der kapillar aufgenommenen Wassermenge und der im Druckverfahren eingebrachten Schutzmittelmenge an querschnittsgleichen Probenabschnitten schwach korrelierende Ergebnisse, die aber vermutlich teilweise auf dem hohen Naßkernanteil in Börs Probematerial beruhen. Es ist somit auch für die Zukunft kein Diagnoseverfahren zu erwarten, mit dem Unterschiede in der Permeabilität von Imprägnierchargen sicher und ausreichend schnell bestimmt werden können. Hinsichtlich des Tränkerfolges bei Weißtanne ist dies aber kaum von praktischem Nachteil, da mit ausreichend hohem Tränkdruck anders als bei Fichte - gute Schutzmittelaufnahmen auch im Kern erzielbar sind. 


\section{Literatur}

Arganbright, D. G.; Wilcox, W. W.: Comparisons of parameters for predicting permeability of white fir. Proceed. Annual Meeting Amer. Wood-Preservers' Assoc.: (1969) 57-62

Bailey, I. W.: The structure of tracheids in relation to the movement of liquids, suspensions, and undissolved gases. In: Physiology of Forest Trees. Roland Press Co., NY (1958) 71-82

Bailey, P. J.; Preston, P. D.: Some aspects of softwood permeability. I. Structural studies with Douglas fir"sapwood and heartwood. Holzforschung 23 (1969) 113-120

Baines, E. F.; Saur, J. M.: Preservative treatment of spruce and other refractory wood. Proceed. Annual Meeting Amer. WoodPreservers' Assoc.: (1985) 136-147

Bauch, J.; Liese W.; Willeitner, H.: Zum Tränkverhalten verschiedener Kiefernarten. Holz Roh-Werkstoff 41 (1983) 339--344

Bellmann, H.: Zur Bedeutung der Holzfeuchte bei der Kesseldrucktränkung von Nadelhölzern. Teil 2. Anatomische und technologische Einflüsse. Holz-Zbl. 143 (1987) 2201-2203

Bör, T. 1991: Imprägnierung von Schnittholz der Tanne (Abies alba Mill.). EMPA St. Gallen, Bericht 23’332:83 S. u. Anhang

Brill, H.; Bock, E.; Bauch, J.: Über die Bedeutung von Mikroorganismen im Holz von Abies alba Mill. für das Tannensterben. Forstwiss. Cbl. 100 (1981) 195-206

Buro, A.; Buro, E. A.: Beitrag zur Kenntnis der Eindringwege für Flüssigkeiten in Kiefernholz. Holzforschung 13 (1959) 71-77

Coté, W. A. Colley Lecture. In search of pathways through wood. Proceed. Annual Meeting Amer. Wood-Preservers' Assoc.: (1990) 97-105

Erickson, H. D.; Balatinecz, J. J.: Liquid flow paths into wood using polymerization techniques - Douglas-Fir and styrene. Forest Products J. 14 (1964) 293-299

Furuno, T.; Goto, T.: Structure of the interface between wood and synthetic polymer. I. On the penetration of MMA monomer into Hinoki. II. The penetration of MMA monomer into wood from five species. J. Japan Wood Res. Soc. 16 (5): 201-208 and 18 (4) $(1970 ; 1972) 159-167$

Humphrey, P. E.; Morrell, J. J.: Distinguishing between refractory and permeable wood at mill production speeds. A possible approach. Proceed. Conf. on Non Destruct. Testing: Pullmann, W. A.: (1988) 269-276

Klein, P.; Bauch, J.; Frühwald A., Naßkerneigenschaften von Tannenholz. Holz-Zbl. 10 (1979) 1465-1466
Liese, W.: Über die Eindringung von öligen Schutzmitteln in Fichtenholz. Holz Roh-Werkstoff 9 (195I) 374-378

Liese, W.; Bauch, J.: On anatomical causes of the refractory behavior of spruce and Douglas fir. J. Inst. Wood Sci. 4 (1967) $3-14$

Moore, G. R.; Kline, D. E.; Blankenhorn, P. E.: Impregnation of wood with a high viscosity epoxy resin. Wood \& Fiber Sci. (15) 3 (1983) 223-234

Passialis, C.; Tsoumis, G.: Characteristics of discoloured and wetwood in fir. IAWA Bul. 5 (1984) 111-120

Pendlebury, A. J.; Coetzee, J.; Sofra, E.; Botha, A.: A new technique to determine solvent penetration in wood. Holzforschung 45 (1990) 205-208

Philips, E. W. J.: Movement of the pit membrane in coniferous woods, with special reference to pressure treatment. Forestry 7 (1933) $109-120$

Schütt, P.: Erste Ansätze zur experimentellen Klärung des Tannensterbens. Schweiz. Z. Forstwes. 132. (1981) 443-452

Sell, J. Präparationsmethode für die einwandfreie Detektion des Hohlraumsystems poröser Baustoffe. Microscop. Acta Suppl. 3 (1979) 9-12

Sell, J.; Kropf, F.; Graf, E.: Oberflächliche Gefügeänderungen bei Fichtenholz durch Druckimprägnierung. Holz. Zbl. 114 (112) (1988) 1605-1606

Sell, J.; Kucera, L.: Schweizer Weißtannenholz - Image-Probleme, Eigenschaften, Förderungsmöglichkeiten. Holz RohWerkstoff 47 (1989) 463-469

Sell, J.; Schnell, G.; Arnold, M.: Qualität gesunder und geschädigter Fichten und Tannen von 6 schweizerischen Standorten. EMPA - Bericht 217 (1988) 112 S.

Siau, J. F. Transport processes in wood. Springer Ser. in Wood Sci: 245 S. 1984

Smulsky, S.; Coté, W. A.: Penetration of wood by a water-borne alkyd resin. Wood Sci. Technol. 18 (1984) 59-75

Trenard Y.; Gueneau P.: Penetration pathways of liquid gallium in wood seen by scanning electron microscopy. Wood \& Fiber Sci. 16 (3) (1984) 403-410

Venturio, J. A.; Arganbright, D. G.: Testing the predictability of preservative treatment of wood. Holzforschung 33 (1979) $23-27$

Wardrop, A. B.; Davies, G. W.: Morphological factors relating to the penetration of liquids into wood. Holzforschung 15 (1961) $130-141$

Willeitner, H.: Tränkbarkeit verschiedener Tannenarten. Mitt. Bundesforsch. Forst- u. Holzwirtschaft 93 (1973) 225-232

\section{Berichtigung:}

Page 296: Equation (2) of the article "Non-destructive wood density distribution measurements using computed tomography" by O. Lindgren et al. should be written

CT-number $=1000 \times\left(\mu_{x}-\mu_{\text {water }}\right) / \mu_{\text {water }}$ 